\title{
Effect of Small Pt Doping on the Electronic Structure of NiMnSb Half-Heusler Alloys
}

\author{
M. Pugaczowa-Michalska* \\ Institute of Molecular Physics, Polish Academy of Science \\ Smoluchowskiego 17, 60-179 Poznań, Poland
}

(Received September 17, 2002; in final form February 10, 2003)

\begin{abstract}
The effect of small $\mathrm{Pt}$ doping on the electronic and magnetic properties of NiMnSb compound were studied using the spin-polarised tight-binding linear muffin-tin orbital method. The electronic structure of half-Heusler alloys depends on the relative arrangement of the atoms in the unit cell, changing from half-metal to metal. The local ordering drastically influences the value of the band gap. The self-consistent band calculations were performed for the experimental lattice constant and for the lattice parameter estimated from the minimum of the total energy.
\end{abstract}

PACS numbers: 71.20.-b, 71.20.Be, 75.50.-y

\section{Introduction}

In recent years the magnetic and transport properties of the half-Heusler alloy NiMnSb have been actively studied [1-13]. The significant interest for NiMnSb is due to its predicted half-metallic behaviour from the band structure calculation [14]. In the terms of electronic structure, it means that the majority spin electrons are in the conduction band, whereas the minority spin electrons are in the semiconducting band. Consequently, the conduction electrons are 100\% spin-polarised at the Fermi level. This feature of NiMnSb leads to investigations of larger amplitudes of giant magneto-resistance (GMR) [3-7] and a peculiarity of the magneto-optical properties $[8,10,11]$.

*e-mail: maria@ifmpan.poznan.pl 
The half-metallic behaviour of NiMnSb is well established experimentally in the bulk. The magnetic moment is measured by neutron experiments $\left(4 \mu_{\mathrm{B}}\right)[12,13]$ in agreement with the expected integer value of the moment for half-metallic ferromagnet. Spin-polarised positron-annihilation [15, 16] and infrared absorption [17] gave $100 \%$ spin-polarisation at the Fermi level. Very close to $100 \%$ polarisation was observed at the Fermi level using the spin-polarised inverse photoemission in NiMnSb [5].

The half-metallic band structure for NiMnSb was confirmed by numbers of studies of the electronic structure using the Korringa-Kohn-Rostoker method (KKR) $[15,18,19]$ and the linear muffin-tin orbital method (LMTO) [20, 21]. In [18] the influence of the atomic disorder on the half-metallic property of NiMnSb was pointed out. It confirmed that the disorder of the crystallographic structure can lead to the reduction of the $100 \%$ spin-polarisation in NiMnSb.

Some of experimental evidences (such as the Hall effect, the magnetoresistance, the termopower) [1, 22] suggest that the NiMnSb alloy may not be half-metallic ferromagnet above $80 \mathrm{~K}$. The changes in electronic properties happen near $80 \mathrm{~K}$ with a very good atomic order in samples. The anomalies, which appeared in various physical properties of NiMnSb near $80 \mathrm{~K}$ have been explained in the terms of a "crossover" from a half-metallic state to a normal metallic ferromagnet [22]. The results of magnetic circular dichroism measurements [23] of $\mathrm{NiMnSb}$ show that a dramatic increase in the Mn and $\mathrm{Ni}$ moments occurs below the "crossover" transition temperature of about $80-100 \mathrm{~K}$.

In the present paper, we have investigated the change of the spin-polarised electronic structure of NiMnSb during small Pt doping and detailed relationship between relative arrangement of the atoms in the unit cell and the value of the band gap, which is the characteristic parameter of electronic structure of half-metallic materials. The magnetic moments were calculated for different distributions of $\mathrm{Pt}$ atoms in the supercell.

The studied $\mathrm{Ni}_{0.875} \mathrm{Pt}_{0.125} \mathrm{MnSb}$ compound crystallises into the $C 1_{b}$ structure (space group $F \overline{4} 3 m$ ) [24]. This noncentrosymmetric crystal structure (shown in Fig. 1) can be obtained from the $\mathrm{Ni}_{2} \mathrm{MnSb}$ ( $L 2_{1}$-type) structure by eliminating one of the fcc Ni sublattices resulting in the tetrahedral coordination of the Sb and

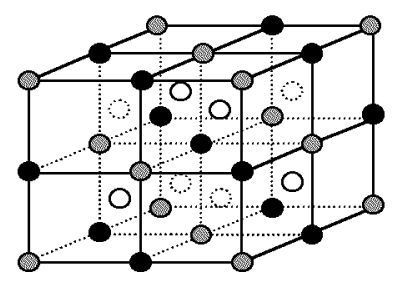
- $\mathrm{A}(\mathrm{Mn})$
$\mathrm{O}(\mathrm{Ni}, \mathrm{Pt})$
- $\mathrm{C}(\mathrm{Sb})$
D (empty site)

Fig. 1. The crystal structure of NiMnSb compound $-C 1_{b}$ structure. 
$\mathrm{Mn}$ atoms by $\mathrm{Ni}$. The electronic structure of $\mathrm{Ni}_{0.875} \mathrm{Pt}_{0.125} \mathrm{MnSb}$ alloy has been calculated for the supercell structure which included a $2 \times 2 \times 2$ primitive cell, i.e. 32 positions per cell. Eight of them are occupied by Mn atoms (A position). Eight of $\mathrm{Sb}$ atoms are in $\mathrm{C}$ positions. The $\mathrm{B}$ positions are occupied by $\mathrm{Ni}$ or $\mathrm{Pt}$ atoms; the $\mathrm{D}$ sites remain unoccupied. In the study of an influence of the local ordering on band gap, we also considered additional distinct structures obtained by interchanging the $\mathrm{Ni}$ or Pt atoms into the empty sites.

According to experimental data [24] the lattice parameter was assumed to be nearly linear dependent on concentration of the Pt atoms in NiMnSb and for studied $\mathrm{Ni}_{0.875} \mathrm{Pt}_{0.125} \mathrm{MnSb}$ the lattice parameter was estimated as $a=0.595 \mathrm{~nm}$. The theoretical lattice parameter corresponding to the minimum of the total energy was obtained too.

\section{Method of calculations}

The electronic band structures and the magnetic moments for $\mathrm{Ni}_{0.875} \mathrm{Pt}_{0.125} \mathrm{MnSb}$ alloy with $C 1_{b}$-type structure (Fig. 1 ) were carried out using the self-consistent spin-polarised TB-LMTO method in the atomic sphere approximation (ASA) [25, 26].

In the atomic sphere approximation the Wigner-Seitz (W-S) cell is replaced by the sphere with the radius $S_{\mathrm{av}}$ which is determined by the following condition:

$$
S_{\mathrm{av}}=a\left(\frac{3}{4 \pi N}\right)^{1 / 3}
$$

where $a$ denotes the lattice parameter and $N$ is the number of atoms in a cell. The values of the Wigner-Seitz radii for the components were estimated from the following relation:

$$
\sum_{n}\left(\frac{S_{n}}{S_{\mathrm{av}}}\right)^{3}=N
$$

where the summation is over all types of atoms in the cell and $S_{n}$ is the atomic radius. For the $C 1_{b}$ structure one empty sphere per formula unit was introduced in the interstitial region in order to reduce the overlap between neighbouring atomic spheres.

The exchange-correlation potential used in these calculations is the functional of von Barth and Hedin [27]. All the total energy carries out for above $500 k$-points in the irreducible Brillouin zone. The initial atomic configurations for each atom were taken as in the periodic table of elements.

The supercell with 32 atoms is taken into consideration. It was obtained by the extension into the three dimensional space of the basic positions of $C 1_{b}$ structure. The supercell was divided into two main sublattices (the first one is formed by A and C positions; the second one is built by B and D positions, Fig. 1). The occupations of the first sublattice, which consists of 16 sites with $\mathrm{Mn}$ and $\mathrm{Sb}$ 
atoms, remain unchangeable during our calculations. For the second sublattice, we have also the same number of sites, among which only eight are occupied by seven $\mathrm{Ni}$ and one Pt. For the rest eight positions, we put empty spheres, as atoms with zero nuclear charge. For $\mathrm{Ni}_{0.875} \mathrm{Pt}_{0.125} \mathrm{MnSb}$, the spin polarised band calculations have been performed in three different crystallographic arrangements of $\mathrm{Ni}$ and $\mathrm{Pt}$ atoms. In the first case (a) we put these atoms only in B positions, so that we have seven kinds of atoms and three different kinds of empty spheres in the supercell. In the second (b) arrangement of occupation of the second sublattice we move one of $\mathrm{Ni}$ atoms from $\mathrm{B}$ to $\mathrm{D}$ position. In the third arrangement $(\mathrm{c})$ the $\mathrm{Pt}$ atom is displaced from $\mathrm{B}$ to $\mathrm{D}$ position. For both (b) and (c) cases there are 13 kinds of atoms and 5 kinds of empty spheres in the supercell.

\section{Results}

Our ab initio calculation has shown that the ground state of small Pt-doping of NiMnSb is ferromagnetic for all presented cases. Figure $2 \mathrm{a}-\mathrm{c}$ presents the electronic structures calculated at an experimental lattice parameter. The half-metallic

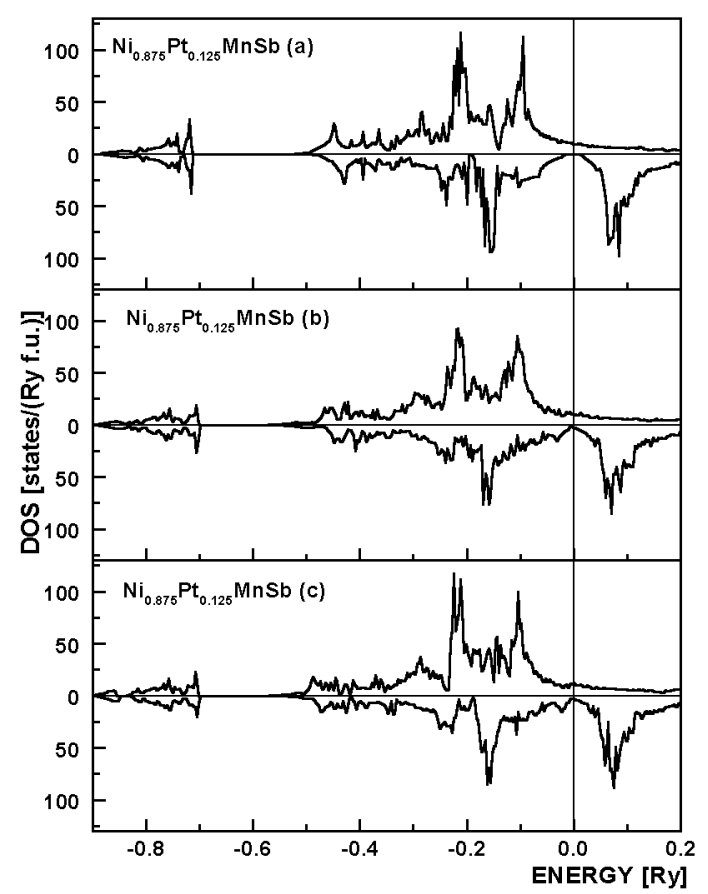

Fig. 2. The electronic density of states for $\mathrm{Ni}_{0.875} \mathrm{Pt}_{0.125} \mathrm{MnSb}$ for three arrangements of atoms in the supercell: (a) The $\mathrm{Ni}$ and $\mathrm{Pt}$ atoms occupy the positions as in $C 1_{b}$ cell; (b) One of Ni atoms occupies the vacancy position; (c) The Pt atom occupies the vacancy position. The vertical line is the Fermi level. 
electronic structure was found in the $\mathrm{Ni}_{0.875} \mathrm{Pt}_{0.125} \mathrm{MnSb}$ with the well-ordered $C 1_{b}$ structure (case (a)). The energy gap at the Fermi level is observed for minority spin carriers only for the case (a). The value of this gap $(\Delta E=0.029 \mathrm{Ry})$ is smaller than the value of the energy gap in pure $\mathrm{NiMnSb}$ alloy $(\Delta E=0.0303 \mathrm{Ry}$ ). The width of the gap of $\mathrm{NiMnSb}$ is in good agreement with the experimental estimated value of the gap [17]. The previous ab initio calculations [21, 28] show that the Fermi level is at the middle of the gap for NiMnSb and the left edge of the gap for PtMnSb. In our calculation for $\mathrm{Ni}_{0.875} \mathrm{Pt}_{0.125} \mathrm{MnSb}$ the shift of the Fermi level into left is not visible yet. The energy gap arises essentially from the hybridisation between the lower energy $d$-states coming from Ni, Pt atoms and the higher energy $d$-states of $\mathrm{Mn}$ atom leading to the formation of bonding and antibonding bands with the gap between them. We observed that the bonding $d$-states are mainly from $\mathrm{Ni}$ while the unoccupied antibonding $d$-states are mainly from Mn (Fig. 3). The total density of states of $\mathrm{Ni}_{0.875} \mathrm{Pt}_{0.125} \mathrm{MnSb}$ is split into two parts. The part of the density of states lying below the gap consists of hybridised $d$-states of transition

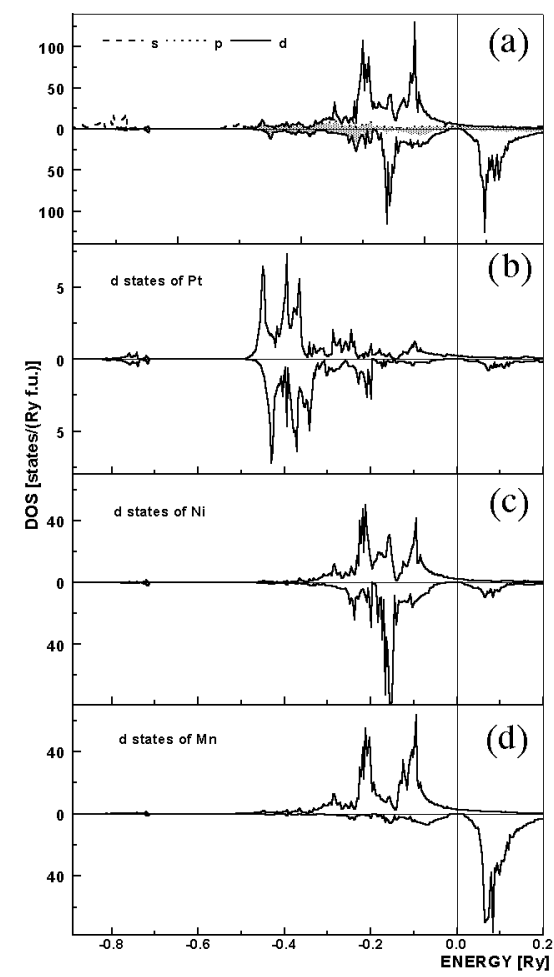

Fig. 3. The densities of states of $\mathrm{Ni}_{0.875} \mathrm{Pt}_{0.125} \mathrm{MnSb}$ for: (a) s-states (dashed line), $p$-states (dotted line, shaded), $d$-states (solid line); (b) $d$-states of Pt atom; (c) $d$-states of $\mathrm{Ni}$ atom; (d) $d$-states of $\mathrm{Mn}$ atom calculated in the $C 1_{b}$ crystal structure for the experimental lattice parameter. 
metals (Ni, Mn, Pt) and the p-states of Sb. The lowest part of the density of states (below $-0.7 \mathrm{Ry}$ ) mostly comes from the $s$-states of $\mathrm{Sb}$ atoms.

In (b) and (c) cases of arrangement of the supercell the gap completely suppressed and the minority band had the metallic character (Fig. 2b, c). The structure with the displacement of $\mathrm{Ni}$ or $\mathrm{Pt}$ atoms to the empty position is not stable when only the bonding states are occupied.

Expecting the changes of the size of the half-metallic gap in the case (a) we carried out the calculations of electronic structure for different lattice parameters. The results plotted in Fig. 4 show that in the region of the lattice parameter close to the experimental one $(0.594 \leq a \leq 0.598 \mathrm{~nm})$ the size of gap has the fixed value $(0.0293 \mathrm{Ry})$, then the value of the gap decreases for a larger value of the lattice parameter. The energy gap shifts above the Fermi level at $a=0.613 \mathrm{~nm}$ and higher values of the lattice parameter. Therefore, the density of states is not zero at the Fermi level (Fig. 5). In the half-metallic region $(0.594 \leq a<0.613 \mathrm{~nm})$ the total magnetic moment of $\mathrm{Ni}_{0.875} \mathrm{Pt}_{0.125} \mathrm{MnSb}$ has an unchanging integer value $\left(m_{\mathrm{tot}}=4.0 \mu_{\mathrm{B}}\right)$. Figure 4 also presents the dependence of the total energy on the lattice parameter. The minimum of the total energy is at $a=0.608 \mathrm{~nm}$. Thus, the theoretical value of the lattice parameter is higher about $2.3 \%$ than the experimental one.

To estimate the nonlocal effects of the value on the gap we additionally calculated the electronic structure of $\mathrm{Ni}_{0.875} \mathrm{Pt}_{0.125} \mathrm{MnSb}$ for the experimental lattice parameter with the Langreth-Mehl-Hu nonlocal exchange-correlation corrections [29]. The value of the gap reduces by $0.005 \mathrm{Ry}$.



Fig. 4. The dependences of relative total energy $\Delta E=E-E_{0}[\mathrm{mRy} / \mathrm{atom}]$ and the size of the half-metallic energy gap on the lattice parameter. The triangle points present the dependence of the total energy. The square points present the size of the energy gap. 


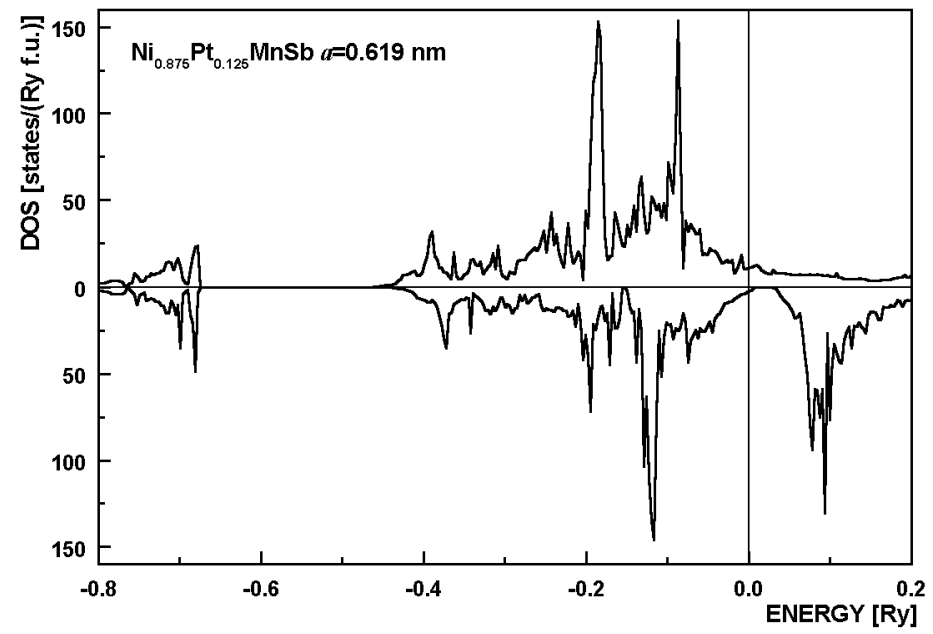

Fig. 5. The densities of states of $\mathrm{Ni}_{0.875} \mathrm{Pt}_{0.125} \mathrm{MnSb}$ for the first arrangement of the supercell at $a=0.619 \mathrm{~nm}$.

The ground state magnetic properties are similar for $\mathrm{Ni}_{0.875} \mathrm{Pt}_{0.125} \mathrm{MnSb}$ in the three arrangements of the supercell. The main contribution to the total magnetic moment comes from the Mn atoms ( $m_{\mathrm{Mn}}=3.58 \div 3.88 \mu_{\mathrm{B}}$ ), which have their minority $d$-band completely shifted above the Fermi level (for example Fig. 3 — the fourth panel). The $3 d$-bands of $\mathrm{Ni}$ are far from the Fermi level and almost

TABLE

The total and local magnetic moments in $\mu_{\mathrm{B}}$ for $\mathrm{NiMnSb}$ and $\mathrm{Ni}_{0.875} \mathrm{Pt}_{0.125} \mathrm{MnSb}$ at the experimental lattice parameter.

\begin{tabular}{|c|c|c|c|c|c|c|}
\hline & $m_{\text {tot }}$ & $m_{\mathrm{Mn}}$ & $m_{\mathrm{Ni}}$ & $m_{\mathrm{sb}}$ & Empty & $m_{\mathrm{Pt}}$ \\
\hline \multirow[t]{2}{*}{$\mathrm{NiMnSb}$} & 4.00 & 3.73 & 0.29 & -0.06 & 0.04 & - \\
\hline & $4.00^{a}$ & $3.76^{a}$ & $0.25^{a}$ & $-0.07^{a}$ & $0.05^{a}$ & \\
\hline \multirow[t]{10}{*}{$\mathrm{Ni}_{0.875} \mathrm{Pt}_{0.125} \mathrm{MnSb} \quad$ (a) } & 4.00 & 3.80 & 0.28 & -0.07 & 0.04 & 0.13 \\
\hline & & 3.70 & 0.29 & -0.05 & & \\
\hline & 3.94 & 3.70 & 0.22 & -0.09 & 0.00 & 0.12 \\
\hline & & 3.81 & 0.24 & -0.06 & 0.04 & \\
\hline & & 3.73 & 0.27 & -0.05 & 0.05 & \\
\hline & & 3.82 & 0.03 & -0.08 & & \\
\hline & 3.90 & 3.58 & 0.26 & -0.06 & 0.04 & 0.003 \\
\hline & & 3.78 & 0.23 & -0.10 & 0.05 & \\
\hline & & 3.73 & 0.22 & -0.09 & 0.06 & \\
\hline & & 3.88 & 0.27 & & 0.00 & \\
\hline
\end{tabular}

\footnotetext{
${ }^{a}$ Reference [21].
} 
filled both spin directions resulting in a small magnetic moment of $m_{\mathrm{Ni}}=0.03 \div$ $0.28 \mu_{\mathrm{B}}$. The Pt moment $\left(m_{\mathrm{Pt}}=0.003 \div 0.13 \mu_{\mathrm{B}}\right)$ is quite small. The small moment at $\mathrm{Sb}$ atom $\left(m_{\mathrm{Sb}}=-0.05 \div-0.10 \mu_{\mathrm{B}}\right)$ couples antiferromagnetically to the $\mathrm{Mn}$ magnetic moments. The values of local magnetic moment for the three cases of occupation $(\mathrm{a}-\mathrm{c})$ and for all kinds of crystallographic inequality positions in the supercell are presented in the Table. The smallest magnetic moment on $\mathrm{Ni}\left(0.03 \mu_{\mathrm{B}}\right)$ and on $\mathrm{Pt}\left(0.003 \mu_{\mathrm{B}}\right)$ is a consequence of a change of the second neighbours for these atoms. The environment of $\mathrm{Ni}$ and $\mathrm{Pt}$ atoms in these cases consists of four $\mathrm{Mn}$ atoms and four $\mathrm{Sb}$ atoms as the first nearest neighbours and five $\mathrm{Ni}$ atoms and one empty sphere as the second neighbours. In the other positions, the $\mathrm{Ni}$ and $\mathrm{Pt}$ atoms are surrounded by four $\mathrm{Mn}$ atoms and four $\mathrm{Sb}$ atoms as the first nearest neighbours and five empty spheres and one $\mathrm{Ni}$ (or $\mathrm{Pt}$ ) atom as the second neighbours. The total magnetic moment is integer only for the exactly half-metallic case (a) of arrangement of atoms in the supercell $\left(m_{\mathrm{tot}}=4.00 \mu_{\mathrm{B}}\right)$. In the cases (b) and (c) the total magnetic moment is smaller than $4 \mu_{\mathrm{B}}$.

\section{Conclusions}

In the present work, the effect of the small $\mathrm{Pt}$ doping on the electronic structure of NiMnSb half-Heusler alloy was studied. The spin-polarised TB-LMTO calculations have shown that the electronic structure of $\mathrm{Ni}_{0.875} \mathrm{Pt}_{0.125} \mathrm{MnSb}$ alloys depends on the relative arrangement of the atoms in the unit cell. The displacement of $\mathrm{Ni}$ or $\mathrm{Pt}$ atom from the sites in the well-ordered $C 1_{b}$ structure to the empty sites can change the property of electronic structure from half-metallic to metallic. In the case of displacement of some atoms ( $\mathrm{Ni}$ or $\mathrm{Pt}$ ) to the empty positions, the gap at the Fermi level is suppressed. The reduction of the total magnetic moment is caused by disorder induced by minority-spin states in this case. Similar result was obtained for pure NiMnSb for different types of atomic disorder [18, 19] using the layer KKR method with coherent potential approximation. The increase in the lattice parameter also influences the value of the band gap. We observed the stable size of the gap nearly the experimental lattice parameter, as well as the shift of the energy gap above the Fermi level at $a=0.613 \mathrm{~nm}$ and at the higher values. It causes a disappearance of the pure half-metallic property of ordered $C 1_{b}$ structure $\mathrm{Ni}_{0.875} \mathrm{Pt}_{0.125} \mathrm{MnSb}$. The similar dependence of the size of the energy gap on the lattice parameter was obtained for PtMnSb in [21].

It has also been found that the magnetic moments on $\mathrm{Ni}$ and $\mathrm{Pt}$ atom strongly depend on the presence of the maximum number of atoms in the second nearest neighbour shell. The smallest values of magnetic moments (on the $\mathrm{Ni}$ and $\mathrm{Pt}$ atoms) are obtained if there are five $\mathrm{Ni}$ atoms in the second nearest neighbour shell. If the number of atoms in the second nearest neighbour shell is only one ( $\mathrm{Ni}$ or $\mathrm{Pt})$, the magnetic moment is more than $0.22 \mu_{\mathrm{B}}$. 


\section{References}

[1] C. Hodequin, J. Pierre, R. Currat, J. Magn. Magn. Mater. 162, 75 (1996).

[2] J.S. Moodera, D.M. Mootoo, J. Appl. Phys. 76, 6101 (1994).

[3] C.T. Tanaka, J. Nowak, J.S. Moodera, J. Appl. Phys. 81, 5515 (1997).

[4] C. Hodequin, J.P. Nozieres, J. Pierre, J. Magn. Magn. Mater. 183, 225 (1998).

[5] D. Ristoui, J.P. Nozieres, C.N. Borca, T. Komesu, H.-K. Jeong, P.A. Dowbern, Europhys. Lett. 49, 624 (2000).

[6] J.A. Caballero, A.C. Reilly, Y. Hao, J. Bass, W.P. Pratt Jr., F. Petroff, J.R. Childress, J. Magn. Magn. Mater. 198-199, 55 (1999).

[7] R. Skomsky, T. Komesu, C.N. Borca, H.-K. Jeong, J. Appl. Phys. 89, 7275 (2001).

[8] J.A. Caballero, W.J. Geerts, F. Petroff, J.-U. Thiele, D. Weller, J.R. Childress, J. Magn. Magn. Mater. 177-181, 1229 (1998).

[9] M.V. Yablonskikh, V.I. Grebennikov, Yu.M. Yarmoshenko, E.Z. Kurmaev, S.M. Butorin, L.-C. Duda, C. Sathe, T. Kaambre, M. Magnuson, J. Nordgren, S. Plogmann, M. Neumann, Solid State Commun. 117, 79 (2001).

[10] X. Gao, J.A. Woollam, R.D. Kirby, D.J. Sellmyer, C.T. Tanaka, J. Nowak, J.S. Moodera, Phys. Rev B 59, 9965 (1999).

[11] J.F. Bobo, P.R. Jonson, M. Kautzky, F.M. Mankoff, J. Appl. Phys. 81, 4164 (1997).

[12] M.J. Otto, H. Feil, R.A. van Woerden, J. Wijngaard, P.J. Van der Valk, C.F. van Brugger, C. Haas, J. Magn. Magn. Mater. 70, 33 (1987).

[13] C. Hordequin, E. Levievre-Berna, J. Pierre, Physica B 236, 603 (1997).

[14] R.A. de Groot, F.M. Mueller, P.G. van Engen, K.H.J. Buschow, Phys. Rev. Lett. 50, 2024 (1983).

[15] K.E.H.M. Hanssen, P.E. Mijnarends, Phys. Rev. B 34, 5009 (1986).

[16] K.E.H.M. Hanssen, P.E. Mijnarends, L.P.M.M. Rabou, K.H.J. Buschow, Phys. Rev. B 42, 1533 (1990).

[17] M.M. Kirillova, A.A. Makhnev, E.I. Shreder, V.P. Dyakina, N.B. Gorina, Phys. Status Solidi B 187, 231 (1995).

[18] D. Orgassa, H. Fujiwara, T.C. Schulthess, W.H. Butler, Phys. Rev. B 60, 13237 (1999)

[19] D. Orgassa, H. Fujiwara, T.C. Schulthess, W.H. Butler, J. Appl. Phys. 87, 5870 (2000)

[20] E.T. Kulatov, I.I. Mazin, J. Phys., Condens. Matter 2, 343 (1990).

[21] S.J. Youn, B.I. Min, Phys. Rev. B 51, 10436 (1995).

[22] C. Hodequin, D. Ristoui, L. Ranno, J. Pierre, Eur. Phys. J. B 16, 287 (2000).

[23] C.N. Borca, T. Komesu, H.-K. Jeong, P.A. Dowben, D. Ristoui, C. Hodequin, J.P. Nozieres, J. Pierre, S. Stadler, Y.U. Idzerda, Phys. Rev. B 64, 52409 (2001).

[24] M. Masumoto, K. Watanabe, Trans. Jpn. Inst. Met. 17, 588 (1976).

[25] O.K. Andersen, O. Jepsen, Phys. Rev. Lett. 53, 2571 (1984). 
[26] O.K. Andersen, O. Jepsen, M. Sob, in: Electronic Structure and its Applications, Ed. M. Yuassoff, Springer, Berlin 1987, p. 2.

[27] U. von Barth, L. Hedin, J. Phys. C 5, 1629 (1972).

[28] I. Galanakis, S. Ostatin, M. Alouani, H. Dreysse, J.M. Wills, Phys. Rev. B 61, 4093 (2000).

[29] C.D. Hu, D.C. Langreth, Phys. Scr. 32, 391 (1985). 